\title{
The Impact of Crustal Magnetic Fields on the Thermal Structure of the Martian Upper Atmosphere
}

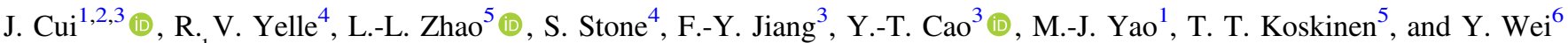 \\ ${ }^{1}$ School of Atmospheric Sciences, Sun Yat-sen University, Zhuhai, Guangdong 519082, People's Republic of China \\ ${ }^{2}$ Lunar and Planetary Science Laboratory, Macau University of Science and Technology, Macau, People's Republic of China \\ ${ }^{3}$ Key Laboratory of Lunar and Deep Space Exploration, National Astronomical Observatories, Chinese Academy of Sciences, \\ Beijing 100012, People's Republic of China \\ ${ }^{4}$ Lunar and Planetary Laboratory, University of Arizona, Tucson, AZ 85721, USA \\ 5 School of Earth Sciences, University of Chinese Academy of Sciences, Beijing 100049, People's Republic of China

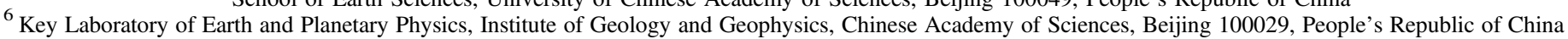 \\ Received 2017 December 7; revised 2018 January 16; accepted 2018 January 17; published 2018 February 2
}

\begin{abstract}
Using the Mars Atmosphere and Volatile Evolution Neutral Gas and Ion Mass Spectrometer data, we investigate the possible impact of crustal magnetic fields on the thermal structure of the Martian upper atmosphere. Our analysis reveals a clear enhancement in temperature over regions with strong crustal magnetic fields during two deep dip campaigns covering the periods of April 17-22 and September 2-8, both in 2015. Several controlling factors, such as solar EUV irradiance, relative atomic $\mathrm{O}$ abundance, and non-migrating tides, do not help to explain the observed temperature enhancement, and a magnetically driven scenario is favored. We evaluate the roles of several heating mechanisms that are likely modulated by the presence of crustal magnetic fields, including Joule heating, ion chemical heating, as well as electron impact heating via either precipitating solar wind electrons or locally produced photoelectrons. The respective heating rates of these mechanisms are substantially lower than the solar EUV heating rate, implying that none of them is able to interpret the observations.
\end{abstract}

Key words: planets and satellites: individual (Mars)

\section{Introduction}

The thermal structure of the dayside Martian upper atmosphere is controlled by solar EUV heating, radiative cooling via $\mathrm{CO}_{2}$ emission at $15 \mu \mathrm{m}$, and thermal conduction (e.g., Bougher \& Roble 1991; Bougher et al. 1999, 2000). The role of solar forcing has been confirmed by a number of authors using the data from the drag experiments made on board Mars Global Surveyor (Forbes et al. 2008; Krasnopolsky 2010), and the data acquired by the Neutral Gas and Ion Mass Spectrometer (NGIMS), the Accelerometer, as well as the Imaging Ultraviolet Spectrograph on board the Mars Atmosphere and Volatile Evolution (MAVEN) spacecraft (Jain et al. 2015; Mahaffy et al. 2015a; Bougher et al. 2017; Zurek et al. 2017).

In addition to solar EUV radiation, solar wind (SW) electron precipitation likely provides an additional heat source for regions with open magnetic field lines. This implies that the temperature in the Martian upper atmosphere is potentially modulated by the strong crustal magnetic fields over the southern hemisphere of the planet (Acuna et al. 1999). Crustal magnetic fields may also affect the local thermal balance either via Joule heating (Vasyliunas \& Song 2005) or via trapping of photoelectrons (Harada et al. 2016). The latter occurs through the magnetic field control of the route along which the photoelectron energy is degraded via collisions with ambient neutrals. In addition, crustal magnetic fields likely affect local plasma distribution (Andrews et al. 2014) and accordingly local heating via exothermic ion chemistry (e.g., Fox 1988). Search for the impact of crustal magnetic fields on the thermal structure of the dayside Martian upper atmosphere was attempted based on the dayglow emission data acquired by the Mars Express (MEx) Spectroscopy for the Investigation of the Characteristics of the Atmosphere of Mars (SPICAM), but no firm conclusion was reached (Leblanc et al. 2006; Stiepen et al. 2015).

At the nightside, the thermal structure of the Martian upper atmosphere is less constrained by observations (Schofield et al. 1997). Analysis of the Mars Pathfinder Atmospheric Structure Investigation data yielded a peak nightside temperature of $153 \mathrm{~K}$ at $134 \mathrm{~km}$ (Magalhães et al. 1999). Measurements made with several instruments on board MAVEN, such as NGIMS, should be able to provide statistical results on the nightside thermal structure, but these results are not yet available when this article is being prepared. SW electron precipitation likely plays an essential role as the dominant heating mechanism, replacing solar EUV heating at the dayside, as motivated by numerous studies of the nightside ionosphere of Mars, both observationally (e.g., Withers et al. 2012) and theoretically (e.g., Lillis et al. 2009). Energy deposition by precipitating electrons may also be accomplished via the transport of dayside photoelectrons to the nightside under favorable magnetic field configurations (Xu et al. 2016). In both cases, an impact of crustal magnetic fields on nightside temperature is expected.

With the aid of the extensive measurements made by the MAVEN NGIMS instrument, we examine in this study whether the observed temperature variability in the Martian upper atmosphere could be magnetically driven. We describe in Section 2 the procedure used for extracting temperature profiles from the $\mathrm{CO}_{2}$ density data. The temperature variability revealed by these data is then presented in Section 3. Specifically, our analysis reveals that neutral temperature over regions with strong crustal magnetic fields tends to be enhanced over regions without. In Section 4, several mechanisms are proposed to interpret the observations. Finally, we provide concluding remarks in Section 5. 

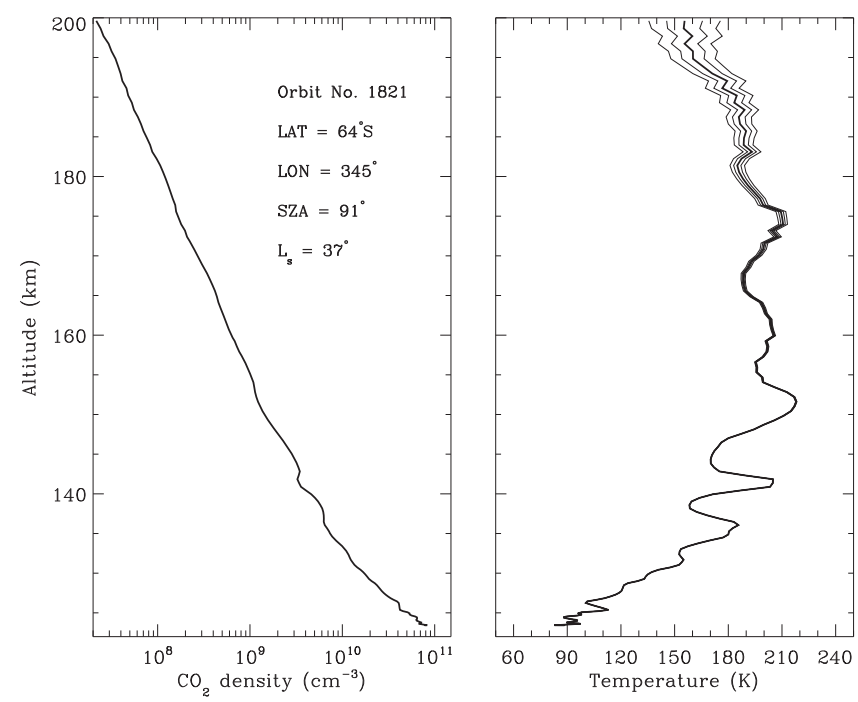

Figure 1. Left: the $\mathrm{CO}_{2}$ density as a function of altitude in the Martian upper atmosphere based on MAVEN NGIMS measurements made during the inbound portion of orbit No. 1821. Right: several temperature profiles derived from the $\mathrm{CO}_{2}$ density data from the same orbit assuming various upper boundary conditions. The thick solid line in the middle corresponds to the case with the optimal upper boundary temperature of $156 \mathrm{~K}$, derived from a Chamberlain model fit to the $\mathrm{CO}_{2}$ density data above $200 \mathrm{~km}$. The thin solid lines cover a range of upper boundary temperatures shifted from the optimal value by $-20,-10,+10$, and $+20 \mathrm{~K}$, respectively.

\section{Extraction of Temperature Profiles}

The present study relies exclusively on the NGIMS level 2 data in the closed source neutral mode publicly available at the MAVEN Science Data Center. ${ }^{7}$ The NGIMS is designed to measure the ambient densities of both neutrals and ions in the Martian upper atmosphere over the mass range of 2-150 Da with a resolution of $1 \mathrm{Da}$ (Mahaffy et al. 2015b). An example is presented in Figure 1 (left) for the $\mathrm{CO}_{2}$ density as a function of altitude from the inbound portion of orbit No. 1821.

The temperature profile shown by the thick solid line in Figure 1 (right) is extracted from the $\mathrm{CO}_{2}$ density data by downward integrating the hydrostatic balance equation, given by

$$
\frac{d}{d r}\left(n k_{\mathrm{B}} T\right)=-m n g
$$

from the exobase, placed at a fixed altitude of $200 \mathrm{~km}$ throughout this study (e.g., Chaffin et al. 2015). In Equation (1), $r$ is the planetocentric radius, $n$ the $\mathrm{CO}_{2}$ number density, $m$ the $\mathrm{CO}_{2}$ molecular mass, $T$ the neutral temperature, $k_{\mathrm{B}}$ the Boltzmann constant, and $g$ the local gravity. Here, we have implicitly assumed that the measured density variation along any MAVEN trajectory is vertical. Meanwhile, we have excluded the outbound $\mathrm{CO}_{2}$ data to minimize contamination by possible reactions on the NGIMS antechamber walls, a wellknown feature that also occurs on other mass spectrometers with a similar design (e.g., Cui et al. 2009). The effects of horizontal variation and wall chemistry have been carefully evaluated based on a comparison between the NGIMS $\mathrm{CO}_{2}$ and Ar data (to be reported in an independent paper) and the crucial

\footnotetext{
https://lasp.colorado.edu/maven/sdc/public/
}

Table 1

Information on Geophysical Parameters for the Four Deep Dip Campaigns (DD1, DD2, DD3, and DD4), Including Latitude (LAT), Solar Zenith Angle (SZA), Local Solar Time (LST), Solar Longitude $\left(L_{\mathrm{s}}\right)$, and $10.7 \mathrm{~cm}$ Solar Radio Index $\left(F_{10.7}\right)$ at 1 au and in SFU $\left(10^{-22} \mathrm{~W} \mathrm{~m}^{-2} \mathrm{~Hz}^{-1}\right)$

\begin{tabular}{lllrrrr}
\hline \hline Campaign & Dates & LAT & SZA & LST & $L_{\mathrm{s}}$ & $F_{10.7}$ \\
\hline DD1 & 2015 & $40^{\circ} \mathrm{N}$ & $108^{\circ}$ & 18.3 & $291^{\circ}$ & 119 \\
& Feb 12-17 & $-44^{\circ} \mathrm{N}$ & & & & \\
DD2 & 2015 & $2^{\circ} \mathrm{S}-6^{\circ} \mathrm{S}$ & $10^{\circ}$ & 11.9 & $329^{\circ}$ & 152 \\
& Apr 17-22 & & & & & \\
DD3 & 2015 Jul 8-14 & $60^{\circ} \mathrm{S}-65^{\circ} \mathrm{S}$ & $110^{\circ}$ & 3.6 & $11^{\circ}$ & 123 \\
DD4 & 2015 Sep 2-8 & $62^{\circ} \mathrm{S}-66^{\circ} \mathrm{S}$ & $91^{\circ}$ & 16.0 & $37^{\circ}$ & 87 \\
\hline
\end{tabular}

results addressed in Section 3 below are found to be unaffected by these effects.

In Figure 1, the optimal upper boundary temperature of $156 \mathrm{~K}$ is obtained by fitting the $\mathrm{CO}_{2}$ density above $200 \mathrm{~km}$ with the traditional Chamberlain model (Chamberlain 1963). We do not choose to determine the upper boundary temperature by assuming zero topside temperature gradient because it could be either positive or negative due to the omnipresence of gravity waves (England et al. 2017; Terada et al. 2017). For a small portion of the orbits considered here that are exclusively at the nightside (see below), publicly available NGIMS $\mathrm{CO}_{2}$ measurements do not extend to altitudes above $200 \mathrm{~km}$, and accordingly we assume a common upper boundary temperature of $140 \mathrm{~K}$ for this specific campaign as motivated by existing measurements reported in Schofield et al. (1997) and Magalhães et al. (1999). For comparison, the thin solid lines in Figure 1 (right) are obtained by adopting a range of upper boundary temperature shifted from the optimal value by -20 , $-10,+10$, and $+20 \mathrm{~K}$, respectively. All temperature profiles converge at sufficiently low altitudes, typically below $170 \mathrm{~km}$.

The $\mathrm{CO}_{2}$ density data from the inbound portion of each MAVEN orbit provide a unique temperature profile from the exobase down to the periapsis. For the purpose of the present study, only the NGIMS measurements made during deep dip (DD) campaigns with periapsis altitudes below $135 \mathrm{~km}$ are included for further analysis. This choice allows an examination of the Martian upper atmosphere as close to the ionospheric peak as possible where the impact of crustal magnetic fields is likely prominent. The sample used here includes the NGIMS data from 65 orbits covering the periods of February 12-17 (hereafter DD1, including 15 orbits), April 17-22 (hereafter DD2, including 14 orbits), July 8-14 (hereafter DD3, including 20 orbits), and September 2-8 (hereafter DD4, including 16 orbits), all in the year of 2015. Detailed information on various geophysical parameters for each campaign is provided in Table 1, including latitude (LAT), solar zenith angle (SZA), local solar time (LST), solar longitude $\left(L_{\mathrm{s}}\right)$, and $10.7 \mathrm{~cm}$ solar radio index $\left(F_{10.7}\right)$ at 1 au and in solar flux units (SFU; $10^{-22} \mathrm{~W} \mathrm{~m}^{-2} \mathrm{~Hz}^{-1}$ ). These parameters cluster tightly around the tabulated mean values. The entire sample spans roughly one third of a Martian year from northern winter to northern spring.

\section{Temperature Variability}

In Figure 2 (left), we compare the mean temperature profiles for all four DD campaigns. The DD3 temperature profile is shown up to an altitude of $170 \mathrm{~km}$ to avoid uncertainties related to the choice of the upper boundary condition (see 

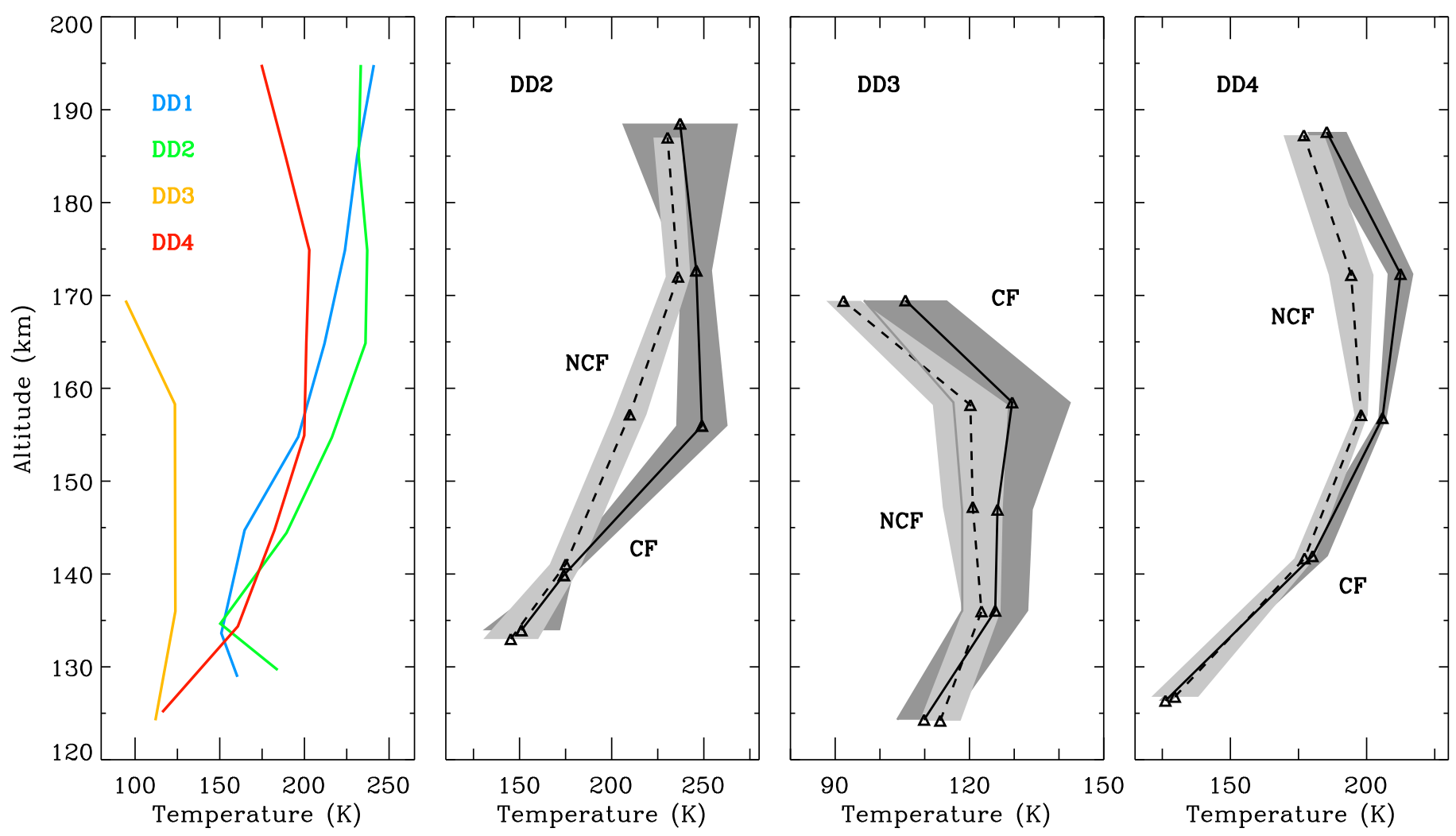

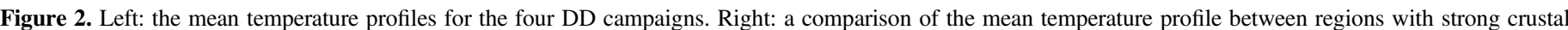

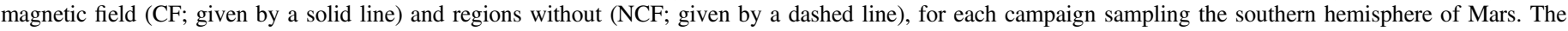

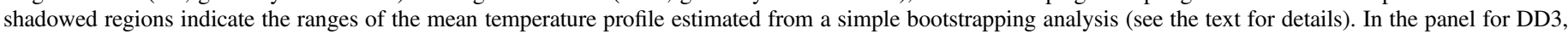

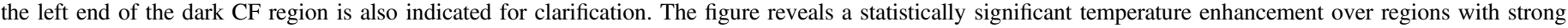
crustal magnetic fields for DD2 and DD4.

Section 2). The exobase temperature, $T_{\text {exo }}$, is estimated to be $230 \mathrm{~K}$ for both DD1 and DD2, $110 \mathrm{~K}$ for DD3, and $190 \mathrm{~K}$ for DD4, respectively. The subsolar temperature of $T_{\text {exo }} \approx 230 \mathrm{~K}$ from DD2 is consistent with our current understanding of the solar cycle and seasonal variations of the Martian upper atmosphere. For comparison, the empirical relation of $T_{\text {exo }} \approx 130.7+1.53 \times F_{10.7}-13.5 \times \cos \left(L_{\mathrm{s}}-85^{\circ}\right) \quad$ given by Forbes et al. (2008) predicts $T_{\text {exo }} \approx 220 \mathrm{~K}$ when the mean values of $F_{10.7}$ and $L_{\mathrm{s}}$ appropriate for DD2 are used (see Table 1). However, a large temperature variability is clearly seen in Figure 2 (left) both near the terminator (DD4) and at the nightside (DD2, DD3). For example, $T_{\text {exo }}$ for DD1 is higher than $T_{\text {exo }}$ for DD3 by as much as $120 \mathrm{~K}$, despite that the two campaigns having a similar SZA coverage and being subject to a similar level of solar EUV irradiance.

For the purpose of the present study, we note from Table 1 that a portion of the available orbits from DD2, DD3, and DD4 cover regions over the southern hemisphere of Mars with strong crustal magnetic fields, allowing their impact on neutral temperature to be scrutinized. Here, we organize all orbits from each campaign into two samples, one characterizing regions with $B_{400}>20 \mathrm{nT}$, hereafter denoted as $\mathrm{CF}$, and the other one with $B_{400}<20 \mathrm{nT}$, hereafter denoted as NCF, where $B_{400}$ is the mean magnetic field strength at $400 \mathrm{~km}$ taken from Lillis et al. (2010). Specifically, CF includes orbit Nos. 1059, 1065, 1071, 1077, and 1081 for DD2; orbit Nos. 1501, 1507, 1511, 1517, 1523, 1529, and 1533 for DD3; and orbit Nos. 1807, 1813, 1823, and 1829 for DD4, respectively. In Figure 2 (right), a comparison of the mean temperature profile between $\mathrm{CF}$ and $\mathrm{NCF}$ is presented for each campaign, showing that the
CF temperature tends to be enhanced over the NCF temperature within a limited altitude range.

To ensure that the above feature does not rely on the observation from any single orbit, we perform a simple bootstrapping analysis by which the data from any orbit within either the CF or NCF sample is discarded successively and the same procedure outlined above is repeated on the reduced sample. The range of the average temperature profiles for each case is indicated by the shadowed region in Figure 2 (right), dark for $\mathrm{CF}$ and light for NCF. The figure reveals that except for DD3, the temperature enhancement is a statistically significant result, reaching $40 \mathrm{~K}$ at about $155 \mathrm{~km}$ for DD2 and reaching $20 \mathrm{~K}$ at about $170 \mathrm{~km}$ for DD4.

Previous attempts to search for the impact of crustal magnetic fields on neutral temperature were made by Leblanc et al. (2006) and Stiepen et al. (2015), both using the dayglow emission data acquired by the MEx SPICAM instrument. Leblanc et al. (2006) reported a very large temperature enhancement of $100 \mathrm{~K}$ over strong crustal magnetic fields, essentially based on a single observation made over the southern hemisphere. Such a conclusion was not confirmed by the more recent study of Stiepen et al. (2015) using a much larger data set covering 10 years of SPICAM observations of dayglow emission in the Martian upper atmosphere. We caution that these early results are based on the scale heights derived from observed emission features of $\mathrm{CO}_{2}^{+}$or $\mathrm{CO}$, and therefore reflect change in mean isothermal temperature rather than the change in temperature profile. Since the modulation of neutral temperature by crustal magnetic fields likely occurs over a limited altitude range as implied by Figure 2 (right), we 
suspect the assumption of an isothermal temperature in previous studies to be the reason for the non-detection of the magnetic field effect.

The thermal structure in the Martian upper atmosphere is controlled by a number of factors, and a careful examination of these factors is required to validate the conclusion that the difference in temperature is indeed related to the difference in magnetic field. Table 1 shows that SZA, $L_{\mathrm{s}}$, and $F_{10.7}$ are constrained within limited ranges for each campaign, implying that the observed temperature difference is unlikely caused by solar cycle, diurnal, and seasonal variations (e.g., Forbes et al. 2008; Jain et al. 2015; Bougher et al. 2017; Zurek et al. 2017). Meanwhile, the difference in relative $O$ abundance is negligible in most cases based on the NGIMS measurements, which implies comparable $\mathrm{CO}_{2}$ cooling between $\mathrm{CF}$ and NCF. The largest difference is seen for $\mathrm{DD} 2$ with the mean $\mathrm{O}$ mixing ratio for $\mathrm{CF}$ depleted by as much as $20 \%$ above $180 \mathrm{~km}$ relative to $\mathrm{NCF}$, but cooling at such high altitudes is dominated by thermal conduction and horizontal advection, rather than $\mathrm{CO}_{2}$ emission (e.g., Bougher et al. 2009). Finally, non-migrating tides, which originate in the lower atmosphere and owe their existence to the topography of Mars (e.g., Forbes \& Hagan 2000), provide a source of longitudinal variability in the Martian upper atmosphere (Lo et al. 2015; Medvedev et al. 2016). Such a possibility is also easily ruled out since the temperature variations produced by topography and crustal magnetization have different longitudinal patterns.

\section{Discussions}

Using the MAVEN NGIMS data from DD2 and DD4, we show in Section 3 that the neutral temperature in the Martian upper atmosphere tends to be enhanced over regions with strong crustal magnetic fields as compared to regions without. Such a feature, if indeed controlled by magnetic field, could be interpreted by several mechanisms including Joule heating, electron impact heating, and ion chemical heating, which we discuss in turn below.

A baseline model for the mean solar EUV energy deposition rate is constructed for each campaign. The NGIMS measurements of both $\mathrm{CO}_{2}$ and $\mathrm{O}$ are adopted in the calculation. The relevant photoabsorption cross-section data are taken from our previous compilation in Cui et al. (2011). For the solar EUV irradiance, we use the appropriate level 3 solar spectral model at Mars, constructed from the Flare Irradiance Spectral ModelMars and calibrated with the MAVEN Extreme Ultraviolet Monitor band irradiance data (Eparvier et al. 2015). The solar energy deposition rate is then multiplied by a constant heating efficiency of 0.2 appropriate for the Martian upper atmosphere (e.g., Fox et al. 2008), to obtain the solar EUV heating rate, hereafter denoted as $Q_{\mathrm{s}}$. The variations of $Q_{\mathrm{s}}$ as a function of altitude are shown with the solid lines in Figure 3 for DD2 (left) and DD4 (right). The figure reveals that CF (red) and NCF (blue) are characterized by a similar $Q_{\mathrm{s}}$ for each campaign.

\subsection{Joule Heating}

A crude estimate of the Joule heating rate, $Q_{\mathrm{J}}$, is made based on the common expression

$$
Q_{\mathrm{J}}=\sigma_{\mathrm{p}} E^{2}
$$
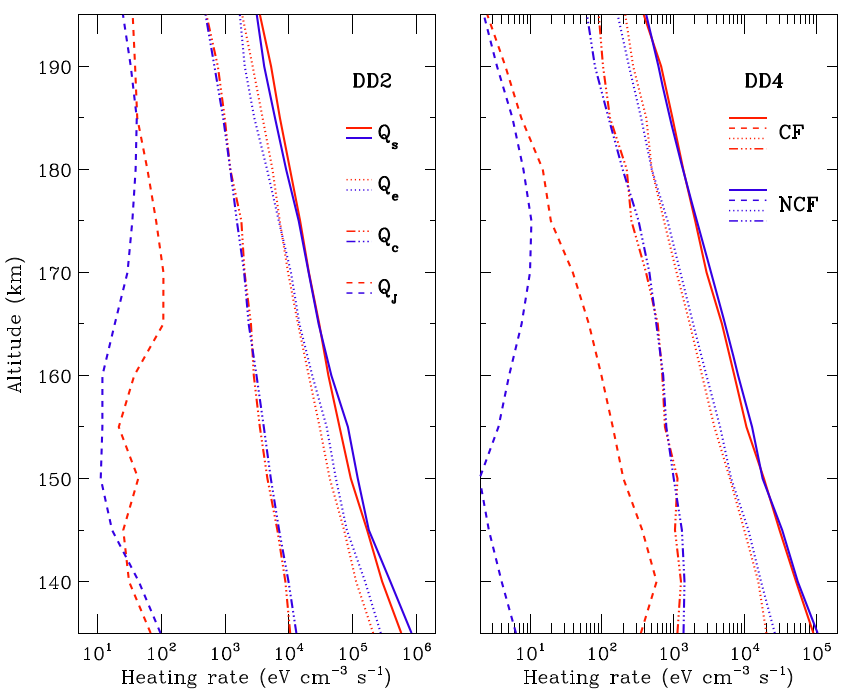

Figure 3. Comparison of various heating rates in the Martian upper atmosphere for DD2 (left) and DD4 (right), including the solar EUV heating rate (solid), the electron impact heating rate (dotted), the ion chemical heating rate (dasheddotted), and the Joule heating rate (dashed). Results for regions with strong crustal magnetic fields (CF; red) and regions without (NCF; blue) are shown separately.

where $\sigma_{\mathrm{p}}$ is the Pedersen conductivity and $E$ is the electric field in the reference frame of the neutrals. $\sigma_{\mathrm{p}}$ could be obtained from known information of the ion-neutral and electronneutral collision frequencies based on the NGIMS $\mathrm{CO}_{2}$ measurements and the electron temperature measurements made by the MAVEN Langmuir Probe and Waves instrument (Ergun et al. 2015), the ion and electron conductivities based on the NGIMS $\mathrm{O}_{2}^{+}$measurements in the open-source ion mode (Benna et al. 2015), and finally the ion and electron gyrofrequencies based on the magnetic field measurements made by the MAVEN Magnetometer (Connerney et al. 2015). Detailed calculations yield $S_{\mathrm{p}}$ below $0.02 \mathrm{~S} \mathrm{~m}^{-1}$ for DD2 and below $0.01 \mathrm{~S} \mathrm{~m}^{-1}$ for DD4, both over regions with strong crustal magnetic fields. These are to be compared with a much larger range of $S_{\mathrm{p}}$ found in regions without strong crustal magnetic fields, which is $(0.02-0.06) \mathrm{S} \mathrm{m}^{-1}$ for DD2 and (0.01-0.07) $\mathrm{S} \mathrm{m}^{-1}$ for DD4. The ranges of $S_{\mathrm{p}}$ quoted above are in harmony with existing results (Opgenoorth et al. 2010).

The electric field in Equation (2) is more difficult to estimate due to the poor knowledge of ion flow velocity in the Martian upper atmosphere. Assuming $200 \mathrm{~m} \mathrm{~s}^{-1}$, which characterizes ionospheric electrodynamics driven by neutral winds (Fillingim et al. 2010), we find a maximum electric field of $E \sim 10-30 \mu \mathrm{V} \mathrm{m}^{-1}$ for DD2 and $E \sim 30-200 \mu \mathrm{V} \mathrm{m}^{-1}$ for DD4 over regions with strong crustal magnetic fields, to be compared with $E \sim 10-20 \mu \mathrm{V} \mathrm{m}^{-1}$ for DD2 and $E \lesssim 5 \mu \mathrm{V} \mathrm{m}^{-1}$ for DD4 over regions without. The corresponding mean Joule heating rates are shown with the dashed lines in Figure 3, red for CF and blue for NCF, which are substantially lower than the respective solar EUV heating rates in all cases. We conclude that, despite a clear magnetically controlled enhancement in Joule heating is implied by the MAVEN data, this heating mechanism falls short by $2-3$ orders of magnitude to account for the observed temperature difference. However, we caution that the near-terminator ion 
flow velocity could be as high as $2 \mathrm{~km} \mathrm{~s}^{-1}$ (Cui et al. 2015). If this is the case, the enhanced Joule heating rate over regions with strong crustal magnetic fields likely reaches several $10^{4} \mathrm{eV} \mathrm{cm}^{-3} \mathrm{~s}^{-1}$, which is a non-negligible fraction of the near-terminator solar EUV heating rate.

\subsection{Electron Impact Heating}

Energetic electrons within the Martian upper atmosphere, including both precipitating SW electrons and photoelectrons, are able to deposit energy and cause heating via inelastic collisions. To evaluate the role of energy deposition from these electrons, we retrieve the calibrated differential energy fluxes from the MAVEN Solar Wind Electron Analyzer level 2 survey data (Mitchell et al. 2016) and calculate the mean electron energy spectrum as a function of altitude for both DD2 and DD4. The electron impact heating rate, $Q_{\mathrm{e}}$, is then calculated via

$$
Q_{\mathrm{e}}=\sum_{\mathrm{i}} \int_{E>W_{\mathrm{i}}} 4 \pi \epsilon \sigma_{\mathrm{i}}(E) N \Phi_{\mathrm{e}}(E) \delta_{\mathrm{i}}(E) d E,
$$

where $E$ is the incident electron energy, $\Phi_{\mathrm{e}}$ is the angularly averaged differential electron flux, $N$ is the $\mathrm{CO}_{2}$ density, $\epsilon$ is the electron impact heating efficiency, and the summation is over all collisional energy loss processes with cross-section, $\sigma_{\mathrm{i}}$, threshold energy, $W_{\mathrm{i}}$, and electron energy loss per collision, $\delta_{\mathrm{i}}$. Various electron impact cross-sections are taken from Cui et al. (2011), including 18 channels of electronic or vibrational excitation and 7 channels of (dissociative) ionization. For excitation processes, $\delta_{\mathrm{i}}$ is taken as $W_{\mathrm{i}}$, whereas for ionization processes, $\delta_{\mathrm{i}}$ includes the contributions from both threshold energy and kinetic energy of the secondary electron, of which the latter is calculated with the aid of the experimental results of Opal et al. (1971). $\epsilon$ is very poorly constrained and likely to be very small (Fox \& Dalgarno 1979). For illustrative purpose, we assume that solar EUV heating at the lower boundary of $130 \mathrm{~km}$ and under subsolar conditions is the sum of photoelectron impact heating and chemical heating via $\mathrm{O}_{2}^{+}$ dissociative recombination (DR; see Section 4.3), as motivated by the modeled thermal balance of the Venusian upper atmosphere (Fox 1988). This yields an electron impact heating efficiency of 0.035 , which is adopted throughout this section. The absolute value of this efficiency is unimportant for the purpose of the present study.

The electron impact heating rates for both DD2 and DD4 are shown with the dotted lines in Figure 3, again red for CF and blue for NCF. The differential electron flux is nearly identical between the two cases. Accordingly, the difference in electron impact heating between CF and NCF is negligible, over both the energy range characteristic of locally produced photoelectrons and the range characteristic of precipitating SW electrons (e.g., Peterson et al. 2016). This implies that neither enhanced SW electron precipitation nor photoelectron trapping is able to account for the NGIMS observations.

\subsection{Ion Chemical Heating}

It is well known that the presence of strong crustal magnetic fields causes localized upwelling in the dayside Martian ionosphere (Andrews et al. 2014 and references therein), which is attributed to the natural outcome of field-aligned plasma diffusion (Matta et al. 2015). This means that various exothermic ion chemical heating rates, which depend on the plasma distribution within the ionosphere, are magnetically controlled. Here, for illustrative purpose, we evaluate the role of both $\mathrm{O}_{2}^{+}$and $\mathrm{CO}_{2}^{+} \mathrm{DR}$ as two of the most important chemical heating channels (Fox 1988), with the respective rate coefficients, branching ratios, as well as exothermicities adapted from Fox (2012) and Fox (2004). The sum of the $\mathrm{O}_{2}^{+}$and $\mathrm{CO}_{2}^{+} \mathrm{DR}$ heating rates is shown with the dashed-dotted line in Figure 3, red for $\mathrm{CF}$ and blue for NCF. For both campaigns, these heating rates are substantially lower than the solar EUV heating rates at all altitudes of interest. Meanwhile, no evidence of enhanced ion chemical heating over strong crustal magnetic fields is seen.

\section{Concluding Remarks}

The thermal structure of the dayside Martian upper atmosphere is well known to be controlled by solar EUV heating, radiative cooling via $\mathrm{CO}_{2}$ emission, and thermal conduction (e.g., Bougher et al. 1999, 2000), but whether or not strong crustal magnetic fields have an impact as well remains unclear (Leblanc et al. 2006; Stiepen et al. 2015). By analyzing the MAVEN NGIMS measurements of $\mathrm{CO}_{2}$ during several DD campaigns that sample the southern hemisphere of Mars, we are able to compare the mean temperature profiles obtained over regions with strong crustal magnetic fields and those obtained over regions without. We observe clear temperature enhancement over crustal magnetic fields for one subsolar campaign (DD2) and another near-terminator campaign (DD4). The temperature enhancement is manifest over a limited altitude range, centered around $155 \mathrm{~km}$ for DD2 and $170 \mathrm{~km}$ for DD4. Several controlling factors, such as solar EUV irradiance, relative atomic $\mathrm{O}$ abundance, and nonmigrating tides, do not help to explain the observed temperature enhancement, and a magnetically driven scenario is favored.

We evaluate the roles of several heating mechanisms that are likely modulated by the presence of crustal magnetic fields by calculating the respective heating rates and comparing them to the solar EUV heating rates. These mechanisms include Joule heating, ion chemical heating, as well as electron impact heating via either precipitating $\mathrm{SW}$ electrons or locally produced photoelectrons. None of these mechanisms is able to interpret the NGIMS observations. Despite this, we caution that the influence of near-terminator Joule heating could be large if the horizontal plasma flow velocity reaches $\gtrsim 1 \mathrm{~km} \mathrm{~s}^{-1}$ as implied by some previous studies of day-to-night transport in the Martian ionosphere (Cui et al. 2015).

Conclusions made in the present study rely exclusively on the NGIMS measurements made during two DD campaigns. Further support is clearly required to confirm the impact of crustal magnetic fields on neutral temperature, as well as to identify the responsible mechanism when more data become available. For this purpose, the data from the MAVEN nominal science orbits may not be very useful since the impact of magnetic field is likely manifest at altitudes below their periapses. This is in contrast to the recent finding that a similar impact on electron temperature is manifest above $200 \mathrm{~km}$ (Flynn et al. 2017).

The authors acknowledge support from the National Science Foundation of China (NSFC) through grants 41525015, 
41774186, and 41504133. J.C. and L.L.Z. are also supported by the Science and Technology Development Fund of Macau SAR (FDCT) through grants 039/2013/A2 and 082/2015/A3.

\section{ORCID iDs}

J. Cui (1) https://orcid.org/0000-0002-4721-8184

L.-L. Zhao (1) https://orcid.org/0000-0002-4299-0490

Y.-T. Cao (1) https://orcid.org/0000-0003-0858-4488

\section{References}

Acuna, M. H., Connerney, J. E. P., Ness, N. F., et al. 1999, Sci, 284, 790

Andrews, D. J., André, M., Opgenoorth, H. J., et al. 2014, JGRA, 119, 3944 Benna, M., Mahaffy, P. R., Grebowsky, J. M., et al. 2015, GeoRL, 42, 8958 Bougher, S. W., Engel, S., Roble, R. G., \& Foster, B. 1999, JGR, 104, 16591 Bougher, S. W., Engel, S., Roble, R. G., \& Foster, B. 2000, JGR, 105, 17669 Bougher, S. W., McDunn, T. M., Zoldak, K. A., \& Forbes, J. M. 2009, GeoRL, 36, L05201

Bougher, S. W., \& Roble, R. G. 1991, JGR, 96, 11

Bougher, S. W., Roeten, K. J., Olsen, K., et al. 2017, JGRA, 122, 1296

Chaffin, M. S., Chaufray, J. Y., Deighan, J., et al. 2015, GeoRL, 42, 9001

Chamberlain, J. W. 1963, P\&SS, 11, 901

Connerney, J. E. P., Espley, J. R., DiBraccio, G. A., et al. 2015, GeoRL, 42,8819

Cui, J., Galand, M., Coates, A. J., Zhang, T. L., \& Müller-Wodarg, I. C. F. 2011, JGRA, 116, A04321

Cui, J., Galand, M., Yelle, R. V., Wei, Y., \& Zhang, S.-J. 2015, JGRA, 120, 2333

Cui, J., Yelle, R. V., Vuitton, V., et al. 2009, Icar, 200, 581

England, S. L., Liu, G., Yiğit, E., et al. 2017, JGRA, 122, 2310

Eparvier, F. G., Chamberlin, P. C., Woods, T. N., \& Thiemann, E. M. B. 2015 , SSRv, 195, 293

Ergun, R. E., Morooka, M. W., Andersson, L. A., et al. 2015, GeoRL, 42, 8846
Fillingim, M. O., Peticolas, L. M., Lillis, R. J., et al. 2010, Icar, 206, 112 Flynn, C. L., Vogt, M. F., Withers, P., et al. 2017, GeoRL, 44, 10812

Forbes, J. M., \& Hagan, M. E. 2000, GeoRL, 27, 3563

Forbes, J. M., Lemoine, F. G., Bruinsma, S. L., Smith, M. D., \& Zhang, X. 2008, GeoRL, 35, L01201

Fox, J. L. 1988, P\&SS, 36, 37

Fox, J. L. 2004, JGRA, 109, A08306

Fox, J. L. 2012, Icar, 221, 787

Fox, J. L., \& Dalgarno, A. 1979, P\&SS, 27, 491

Fox, J. L., Galand, M. I., \& Johnson, R. E. 2008, SSRv, 139, 3

Harada, Y., Mitchell, D. L., Halekas, J. S., et al. 2016, GeoRL, 43, 939

Jain, S. K., Stewart, A. I. F., Schneider, N. M., et al. 2015, GeoRL, 42, 9023 Krasnopolsky, V. A. 2010, Icar, 207, 638

Leblanc, F., Chaufray, J. Y., Lilensten, J., Witasse, O., \& Bertaux, J.-L. 2006, JGRE, 111, E09S11

Lillis, R. J., Fillingim, M. O., Peticolas, L. M., et al. 2009, JGRE, 114, E11009 Lillis, R. J., Purucker, M. E., Halekas, J. S., et al. 2010, JGRE, 115, E07007 Lo, D. Y., Yelle, R. V., Schneider, N. M., et al. 2015, GeoRL, 42, 9057 Magalhães, J. A., Schofield, J. T., \& Seiff, A. 1999, JGR, 104, 8943 Mahaffy, P. R., Benna, M., Elrod, M., et al. 2015a, GeoRL, 42, 8951 Mahaffy, P. R., Benna, M., King, T., et al. 2015b, SSRv, 195, 49 Matta, M., Mendillo, M., Withers, P., \& Morgan, D. 2015, JGRA, 120, 766 Medvedev, A. S., Nakagawa, H., Mockel, C., et al. 2016, GeoRL, 43, 3095 Mitchell, D. L., Mazelle, C., Sauvaud, J.-A., et al. 2016, SSRv, 200, 495 Opal, C. B., Peterson, W. K., \& Beaty, E. C. 1971, JChPh, 55, 4100

Opgenoorth, H. J., Dhillon, R. S., Rosenqvist, L., et al. 2010, P\&SS, 58, 1139 Peterson, W. K., Thiemann, E. M. B., Eparvier, F. G., et al. 2016, JGRA, 121,8859

Schofield, J. T., Barnes, J. R., Crisp, D., et al. 1997, Sci, 278, 1752

Stiepen, A., Gérard, J.-C., Bougher, S., et al. 2015, Icar, 245, 295

Terada, N., Leblanc, F., Nakagawa, H., et al. 2017, JGRA, 122, 2374

Vasyliunas, V. M., \& Song, P. 2005, JGRA, 110, A02301

Withers, P., Fillingim, M. O., Lillis, R. J., et al. 2012, JGRA, 117, A12307

Xu, S., Mitchell, D., Liemohn, M., et al. 2016, GeoRL, 43, 8876

Zurek, R. W., Tolson, R. A., Bougher, S. W., et al. 2017, JGRA, 122, 3798 